\title{
Melatonin supplementation to improve quality of life for elderly cancer patients
}

\author{
Angeline Ginzac ${ }^{1,2 *}$, Emilie Thivat ${ }^{1,2}$ and Xavier Durando ${ }^{1,2}$ \\ ${ }^{1}$ Université Clermont Auvergne, INSERM, U1240 Imagerie Moléculaire et Stratégies Théranostiques, F-63000 Clermont-Ferrand, France \\ ${ }^{2}$ Délégation Recherche Clinique \& Innovation, Centre Jean Perrin, F-63011 Clermont-Ferrand, France
}

\begin{abstract}
The incidence of elderly population living with cancer increases. Maintaining or improving the quality of life (QoL) has become an important goal in the treatment of cancer disease and is even an endpoint in clinical trials. The elderly are underrepresented within these clinical trials and often undertreated. The aged population with cancer is very heterogeneous and has certain characteristics (comorbidities, vulnerability) making the management and assessment of QoL more complex in this population.

Melatonin, a hormone secreted by the pineal gland, regulates various physiological functions and is involved in the initiation of sleep. With age, the secretion of melatonin decreases and thus disrupts circadian rhythm. Circadin ${ }^{\circledR}$ (a prolonged-release form of melatonin) is used in France in the treatment of primary insomnia in person over 55 years old and contributes to improvement of QoL. Melatonin also presents a potential interest in addition to chemotherapy in the treatment of cancer by reducing or preventing certain symptoms (e.g., fatigue, depression) that constitute essential components of QoL. In this context, it seems appropriate to study the impact of supplementation with melatonin during chemotherapy on QoL of elderly patients with metastatic cancer.
\end{abstract}

\section{Introduction}

In oncology, since all anti-cancer drugs have side effects, it is important to consider the benefits and the risks associated with these treatments, especially in the elderly. This population is more vulnerable to toxicities of the treatment and is more susceptible to the side effects [1]. The elderly population is very heterogeneous and treatment of cancer in this population requires knowledge not only of the disease but also of physiological and pathophysiological features associated with aging. Indeed, the presence of geriatric syndromes (e.g., impaired vision and hearing, incontinence, poor nutrition) represents additional important difficulties regarding treatment of the elderly [2]. In addition, comorbidities have an impact on the choice of the treatment (e.g., anthracyclin increasing the risk of cardiotoxicity such as heart failure, cisplatin increasing the risk of renal failure) and lead to polymedication, which contributes to increased chemotherapy toxicity in older patients [1].

This mini review will discuss about the interest of melatonin supplementation during cancer treatment for elderly patients.

\section{Melatonin and elderly patients}

In humans, the pineal gland becomes less functional as age advances and melatonin levels gradually decline over the life span [3]. The causes of the decrease in melatonin secretion are not fully understood at this stage, and several hypotheses have been suggested, such calcification of the pineal gland, or abnormal transmission of the light signal [4]. Another suggested hypothesis is a decrease of melatonin receptors (MT1) in the brain, which is age-related [5].

The melatonin secretion during aging inversely correlates with sleep disturbances. One study has also demonstrated a link between urinary melatonin reduction and poor quality of sleep in the elderly, and delayed sleep phase, identified by comparison with a control group of the same age [6].

\section{Circadian rhythm disruption in cancer patients and quality of life}

Decrease of melatonin secretion conduct to the circadian rhythm disruption. This latter has been correlated with a lower QoL as a whole in a total of 226 patients with metastatic colorectal cancer [7]. Another study indicated that circadian rhythm alterations were associated with poor performance status and liver metastasis in 13 patients with advanced breast cancer [8].

There could be a link between a high rate of cytokines and circadian system disturbance $[9,10]$. Innominato et al. [11] also showed in a study of 96 patients with metastatic colorectal cancer, that disruption of the circadian system was associated with a lower QoL as a whole and in various domains, including social environment.

\section{Melatonin during cancer treatments}

Melatonin prolonged-release $2 \mathrm{mg}$ has received its authorization in the European market (EU/1/07/392/001; CIP 3400938414158) as a monotherapy and should be given two hours prior to sleep and can be used in the short-term treatment of primary insomnia in adults aged 55 and more. The most common side effects reported in trials

${ }^{*}$ Correspondence to: Angeline GINZAC, 58, rue Montalembert, 63011 Clermont-Ferrand, France, Tel: +33 473278005; Fax: +33 473278029; E-mail: Angeline.ginzac@clermont.unicancer.fr

Key words: melatonin, quality of life, elderly patients, cancer treatment

Received: May 10, 2019; Accepted: May 21, 2019; Published: May 25, 2019 
were headache, nasopharyngitis, back pain and arthralgia; they were observed both in Circadin $\odot$ and placebo groups.

A study on 200 patients with chemotherapy resistant metastatic cancer, comparing chemotherapy versus chemotherapy + melatonin at a dose of $20 \mathrm{mg} / \mathrm{d}$ for at least 2 months, showed a significant reduction in chemotherapy-induced toxicities such as asthenia, thrombocytopenia, neurotoxicity and stomatitis. Another study conducted by the same team showed a significant reduction in some cancer-induced symptoms by providing melatonin to 1440 patients: 718 patients treated with supportive care alone, and 722 treated with supportive care + melatonin at $20 \mathrm{mg} / \mathrm{d}$ [12]. The improved symptoms were cachexia; asthenia, anorexia, depressive syndromes, and thrombocytopenia.

Also according to meta-analysis $[13,14]$, the melatonin significantly reduces symptoms such as asthenia, leukopenia, nausea and vomiting, hypotension, and thrombocytopenia. As these symptoms are mostly included in the measurement scales of the QoL, their improvement by melatonin could be translated into patient QoL improvement.

A double-blind trial studied the effect of melatonin supplementation (10 or $20 \mathrm{mg} / \mathrm{d}$ ) on QoL [15]. It reported a better adjusted QoL score (FACT-L) with a slightly significant better score ( 2.69 points, $\mathrm{CI}=0.01$ $5.38, \mathrm{p}=0.049$ ) being found in social wellbeing.

\section{MEQAPAG trial}

According to these data, Jean PERRIN Comprehensive Cancer Center has set up a phase III national multicenter, double-blind, randomized, placebo-controlled trial (NCT02454855). The main objective of the trial is to study the impact of a melatonin supplementation on the quality of life in elderly metastatic cancer patients ( $\geq 70$ years). The oral supplementation will start at the beginning of a new line of treatment for metastatic cancer and will be administered for three months. Patients will take Circadin ${ }^{\star}$ at a dose of $2 \mathrm{mg} /$ day or placebo, according to randomization, 1-2 hours before bedtime. The quality of life will be assessed using the QLQ-C30 questionnaire. Five hundred patients are needed to be recruited in this study and recruitment is ongoing.

\section{Conclusion}

The use of melatonin as a therapeutic strategy seems particularly suitable for elderly patients, a population known to secrete significantly less melatonin. However, to date no studies have been conducted in this population. The elderly population is heterogeneous and complex (comorbidities, general health, drug interactions, dosage adjustment), and maintaining the QoL is a major challenge in the care of elderly patients. In advanced disease, treatment is palliative, with the aim of controlling the disease and pain, limiting toxicities and maintaining the overall maintenance of QoL. It seems appropriate to study the impact of melatonin supplementation during a cytotoxic therapy on QoL in elderly metastatic cancer patient.

\section{Acknowledgment}

MEQAPAG trial received funding from a hospital clinical research program from French Cancer National Institute (PHRC-K13-170).

\section{References}

1. Popa MA, Wallace KJ, Brunello A, Extermann M, Balducci L (2014) Potential drug interactions and chemotoxicity in older patients with cancer receiving chemotherapy. $J$ Geriatr Oncol 5: 307-314. [Crossref]

2. Kane RL, Ouslander JG, Abrase IB (1989) The Is of Geriatric Syndromes. Essentials of Clinical Geriatrics $2^{\text {nd }}$ Edn.

3. Nogueira LM, Sampson JN, Chu LW, Yu K, Andriole G, et al. (2013) Individual variations in serum melatonin levels through time: implications for epidemiologic studies. PLoS ONE 8: e83208. [Crossref]

4. Skene DJ, Swaab DF (2003) Melatonin rhythmicity: effect of age and Alzheimer's disease. Exp Gerontol 38: 199-206. [Crossref]

5. Wu Y-H, Zhou J-N, Van Heerikhuize J, Jockers R, Swaab DF (2007) Decreased MT1 melatonin receptor expression in the suprachiasmatic nucleus in aging and Alzheimer's disease. Neurobiol Aging 28: 1239-1247. [Crossref]

6. Haimov I, Laudon M, Zisapel N, Souroujon M, Nof D, et al. (1994) Sleep disorders and melatonin rhythms in elderly people. BMJ 309: 167. [Crossref]

7. Mormont MC, Waterhouse J, Bleuzen P, Giacchetti S, Jami A, et al. (2000) Marked 24-h rest/activity rhythms are associated with better quality of life, better response, and longer survival in patients with metastatic colorectal cancer and good performance status. Clin Cancer Res 6: 3038-3045. [Crossref]

8. Touitou Y, Lévi F, Bogdan A, Benavides M, Bailleul F, et al. (1995) Rhythm alteration in patients with metastatic breast cancer and poor prognostic factors. J Cancer Res Clin Oncol 121: 181-188. [Crossref]

9. Rich T, Innominato PF, Boerner J, Mormont MC, Iacobelli S, et al. (2005) Elevated serum cytokines correlated with altered behaviour, serum cortisol rhythm, and dampened 24-hour rest-activity patterns in patients with metastatic colorectal cancer. Clin Cancer Res 11: 1757-1764. [Crossref]

10. Innominato PF, Mormont M-C, Rich TA, Waterhouse J, Lévi FA, et al. (2009) Circadian disruption, fatigue, and anorexia clustering in advanced cancer patients: implications for innovative therapeutic approaches. Integr Cancer Ther 8: 361-370. [Crossref]

11. Innominato PF, Focan C, Gorlia T, Moreau T, Garufi C, et al. (2009) Circadian rhythm in rest and activity: a biological correlate of quality of life and a predictor of survival in patients with metastatic colorectal cancer. Cancer Res 69: 4700-4707. [Crossref]

12. Lissoni $\mathrm{P}$ (2002) Is there a role for melatonin in supportive care? Support Care Cancer 10: 110-116. [Crossref]

13. Wang Y, Jin B, Ai F, Duan C, Lu Y, et al. (2012) The efficacy and safety of melatonin in concurrent chemotherapy or radiotherapy for solid tumors: a meta-analysis of randomized controlled trials. Cancer Chemother Pharmacol 69: 1213-1220. [Crossref]

14. Seely D, Wu P, Fritz H, Kennedy DA, Tsui T, et al. (2012) Melatonin as adjuvant cancer care with and without chemotherapy: a systematic review and meta-analysis of randomized trials. Integr Cancer Ther 11: 293-303. [Crossref]

15. Sookprasert A, Johns NP, Phunmanee A, Pongthai P, Cheawchanwattana A, et al (2014) Melatonin in Patients with Cancer Receiving Chemotherapy: A Randomized, Double-blind, Placebo-controlled Trial. Anticancer Res 34: 7327-7337. [Crossref]

Copyright: (C2019 Ginzac A. This is an open-access article distributed under the terms of the Creative Commons Attribution License, which permits unrestricted use, distribution, and reproduction in any medium, provided the original author and source are credited. 